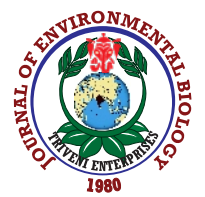

\title{
Assessment of genetic diversity among Egyptian and Saudi chicken ecotypes and local Egyptian chicken breeds using microsatellite markers
}

\author{
A. Sabry ${ }^{1,2 *}$, S. Ramadan ${ }^{3}$, M.M. Hassan ${ }^{1,4}$, A.A. Mohamed ${ }^{1,5}$, A. Mohammedein ${ }^{1}$ and M. Inoue-Murayama ${ }^{6}$ \\ 'Department of Biology, College of Science, Taif University, P.O. Box 11099, Taif-21944, SaudiArabia \\ ${ }^{2}$ Cell Biology Department, National Research Center, 12622, Dokki, Giza, Egypt \\ ${ }^{3}$ Department of Animal Wealth Development, Faculty of Veterinary Medicine, Benha University, Moshtohor, 13736, Egypt \\ ${ }^{4}$ Department of Genetics, Faculty of Agriculture, Menoufia University, 51132, Egypt \\ ${ }^{5}$ Department of Animal Reproduction and AI,National Research Center, 12622, Dokki, Giza, Egypt \\ ${ }^{6}$ Wildlife Research Center, Kyoto University, Kyoto, 606-8203, Japan \\ *Corresponding Author Email : a.sabri@tu.edu.sa
}

\begin{abstract} pure chicken breeds using 14 microsatellite markers.

Methodology: Dataset consisted of two subsets. First subset represented two ecotypes from Saudi Arabia and Egypt. Second dataset consisted birds six Egyptian native chicken strains: Fayoumi (FAY), Dandarawy (DAN), Baladi (BAL), Sinai (Sini), El-Salam (Els) and Golden Montazah (GG), and two commercial strains: White Leghorn (WL) and Rhode Island Red (RIR). fourteen microsatellites markers were utilized to assess DNA polymorphism. Data analyses of the results were carried out using R statistical environment.

Results: The obtained results indicated that number of alleles per locus averaged $11.4 \pm 5.0$. Polymorphic information content was informative (>50\%) for the local breeds, but not for two ecotypes. The observed and expected heterozygosity averaged 0.46 and 0.75 , both ecotypes had the lowest estimates. All breeds showed significant deviation from Hardy-Weinberg equilibrium expectation. The average population differentiation index $\left(F_{\mathrm{ST}}\right)$ was 0.143 , overall heterozygosity deficiency $\left(F_{\mathrm{IT}}\right)$ was 0.156 , and global inbreeding of individuals within breeds $\left(F_{I S}\right)$ was 0.319 .
\end{abstract}

Aim: To assess genetic diversity of two chicken ecotypes from Egypt and Saudi Arabia and compare these ecotypes to six local Egyptian and two exotic

Interpretation: This study revealed that both Egyptian and Saudi ecotypes have an endangered status. Lower genetic distances and lower $F_{\mathrm{ST}}$ values were reported for the Egyptian indigenous breeds. Phylogenetic and principle components showed that both ecotypes were genetically closer to each other when compared with other breeds. It also showed that the Dandarawy native Egyptian chicken breed was genetically the closest breed to both the Egyptian and Saudi ecotypes.

Key words: Chicken, Ecotypes, Genetic diversity, Microsatellites, Phylogenetics

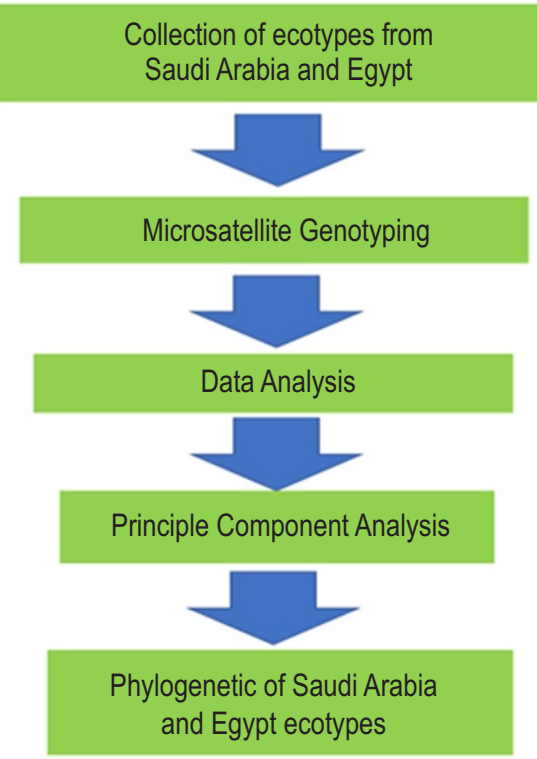

How to cite : Sabry, A., S. Ramadan, M.M. Hassan, A.A. Mohamed, A. Mohammedein and M. Inoue-Murayama: Assessment of genetic diversity among Egyptian and Saudi chicken ecotypes and local Egyptian chicken breeds using microsatellite markers. J. Environ. Biol., 42, 33-39 (2021). 


\section{Introduction}

Despite the significant economic value of indigenous chickens to smallholders in developing countries (Sabry et al., 2017), the existence of these breeds is associated with multiple risk factors (Dwivedi et al., 2007; Muchadeyi et al., 2007; Sanusi and Oseni, 2020). In Egypt, for instance, $90 \%$ of the chicken production is provided by commercial poultry farms (which use imported breeds) of different capacities, with the remaining 10\% provided by small-scale household poultry farms that are abundant in villages and urban areas (Shatokhin et al., 2017). This reliance on commercial imported breeds precludes indigenous chickens from the production system, thus neglecting important genetic resources and exposing the indigenous breeds to the threat of extinction (Hwangbo et al., 2009; Shafey et al., 2017).

The use of ecotypes to characterize genetic structure of indigenous chicken breeds has been studied (Muchadeyi et al., 2007; Sabry et al., 2017) and used as a sampling template in genetic diversity studies (Shafey et al., 2017). Indigenous chickens in Taif governorate, approximately $1.7 \mathrm{~km}$ above sea level, are adapted to harsh environmental conditions and high altitudes. Places at higher elevations generally have extreme environmental conditions, including low oxygen content, reduced partial pressure of oxygen, and daily temperature fluctuations (Rudresh et al., 2015; Huang et al., 2016; Sanusi and Oseni, 2020). These local Saudi chickens are the result of decades of adaptation and domestication, which in turn represent a valuable genetic resource. To the best of our knowledge, the genetic constituents and makeup of the Taif chicken ecotype have not been studied, nor there is reliable information about the adaptation of this ecotype. Osaman et al. (2016) investigated the complete sequence of mitochondrial DNA D-loop to clarify the origin of native Egyptian and Asian chickens. The results revealed that the Egyptian native and West and Central Asian chickens shared a common ancestor as they clustered together in the same clade. These results imply that the Saudi and Egyptian native breeds are genetically close.

The Egyptian poultry production system is rich in local chicken breeds. Based on their morphology, these breeds have been categorized into three categories (Ramadan et al., 2012; Soltan et al., 2018), and are also adapted to hard conditions of Egypt. The first category comprise the pure indigenous breeds Fayoumi and Dandarawy. The second category includes hybrid strains, such as the Baladi and Sinai strains, which were developed by crossing of exotic and Egyptian autochthonous chickens due to ancient trade dispersal and colonization of Egypt. The third group includes genetically improved local strains that originated from crossing local and standardized exotic chicken strains, accompanied by selection for fast growth, such as the ElSalam strain or high egg production, such as the Golden Montazah strain (Abdel-Alah et al., 2017). Advances in molecular technology have provided the opportunity to evaluate genetic variation at DNA level. Microsatellite markers generally have codominant as they are abundant, inheritance, and arbitrarily disseminated in the genome, highly polymorphic and reproducible (Hillel et al., 2003; Shafey et al., 2017). These features reveal that microsatellite markers have been used to assess and characterize genetic diversity in many commercial and indigenous chicken populations (Seo et al., 2013; Abebe et al., 2015; Fathi et al., 2017). Numerous microsatellite markers are available in chickens and have been used to construct linkage maps in many populations (Noneset al., 2005; Sabry et al., 2017).
These markers enable the search for quantitative trait loci and have been effectively used to consider the hereditary connections between and within chicken populations (Hillel et al., 2003; Fathi et al., 2017). On light of the above, this study was conducted to assess genetic diversity of two chicken ecotypes from Egypt and the Kingdom of Saudi Arabia and compare these to six local Egyptian and two exotic pure chicken breeds using 14 microsatellite markers.

\section{Materials and Methods}

Data set: Two subsets were used in the present study. The first dataset comprised two ecotypes from Taif Governorate, Saudi Arabia $(n=25)$, and Ismailia Governorate, Egypt $(n=25)$. These two ecotypes were abbreviated as TA and BAL-ISM. The second dataset consesteted of 281 birds from six Egyptian native brides and two commercial breads. Namely: Fayoumi (FAY, $n=$ 35), Dandarawy (DAN, $n=30$ ), Baladi (BAL, $n=29)$, Sinai (Sini, $n$ $=30$ ), El-Salam (Els, $n=36$ ), and Golden Montazah ( $G G, n=36$ ), and two commercial strains: White Leghorn (WL, $n=42)$, and Rhode Island Red (RIR, $n=43)$.

DNA extraction: Blood samples were collected from two ecotypes for DNA extraction. Genomic DNA was extracted from whole blood using a blood DNA extraction kit (QIAGEN, USA). The quality and quantityof DNA was checked by electrophoresis using a mini-gel and quantified using a NanoDrop 2000C spectrophotometer (Spectronic Genesys, USA). DNA samples were stored at $20^{\circ} \mathrm{C}$.

Microsatellite genotyping: Fourteen microsatellite markers were utilized to assess DNA polymorphism of ten breeds (Table 1). These 14 microsatellite markers were selected based on the reports of International Society of Animal Genetics - Food and Agriculture Organization of the United Nations to investigate the heredity of an assorted variety of chickens (FAO, 2011). Amplification of DNA was performed by polymerase chain reaction in an absolute volume of $50 \mu$ l, with $50 \mathrm{ng}$ of genomic DNA, 10 pmol of fluorescent dye (FAM, VIC, NED, or PET) labeled modified forward primer and unlabeled reverse primer (Applied Biosystems, Virginia, USA), $2.5 \mathrm{mM}$ of each dNTP (GeNet Bio, Seoul, Korea), $10 \times$ reaction buffer (GeNet Bio), and 2.5 units of prime Taq DNA polymerase (GeNetBio, Seoul, Korea).

PCR was performed under following conditions: initial denaturation at $95^{\circ} \mathrm{C}$ for $10 \mathrm{~min}$, followed by 35 cycles of $30 \mathrm{sec}$ of denaturation at $95^{\circ} \mathrm{C}$ for $30 \mathrm{sec}$, annealing at $60^{\circ} \mathrm{C}$ for $30 \mathrm{sec}$, extension at $72^{\circ} \mathrm{C}$, and a final extension at $72^{\circ} \mathrm{C}$ for 10 min using a My-Genie 96 Thermal Cycler (Bioneer, Seoul, Korea). PCR products were initially electrophoresed on a $3 \%$ agarose gel with ethidium bromide to confirm whether they gave a single DNA band under UV light. On clear appearance of band, further genotyping was performed. For microsatellite genotyping, the PCR products were diluted more than 20 times. For genotyping, $1 \mu \mathrm{l}$ of diluted PCR products, $10 \mu \mathrm{l}$ of Hi-Di Formamide (Applied Biosystems, USA), and $0.1 \mu$ l of GeneScan500 LIZ size standard (Applied Biosystems) were denatured for 2 min at $95^{\circ} \mathrm{C}$, and the fragment analysis was performed using a Genetic Analyzer 3130xl (Applied Biosystems). The microsatellite genotypes were analyzed using Gene Mapper v 3.7 (Applied Biosystems).

Data analyses: Data analyses and graphical representation of the results were carried out by $R$ statistical environment ( $R$ Core 
Team, 2020). Fundamental measures of genetic diversity were computed using the adegenet $\mathrm{R}$ statistical package (Jombart, 2008). Phylogenetic analysis was performed by Nei' s distance and population subdivision was examined by Weir and Cockerham's unbiased estimation of Wrights fixation indices $\left(F_{I T}\right.$, $F_{S T}$, and $F_{\text {IS }}$ ) with Population and Evolutionary Genetics Analysis System (pegas) package in R. Null hypothesis was that the estimates were not significant, and the level of significance $(p<0.01)$ was adjusted using Bonferroni corrections. Allelic richness $\left(R_{t}\right)$ was estimated by Hierfstat package in $R$.

\section{Results and Discussion}

In general, two approaches are commonly used to quantify genetic diversity. The first approach utilizes data that describe variation in discrete allelic state, and the second approach utilizes quantitative characters, which leads to different criteria for genetic diversity (Hughes et al., 2008; Shafey et al., 2017). In the present study, the first approach was used. A descriptive overview of the sample size, number of alleles per locus, polymorphic information content (PIC), and expected and observed heterozygosity are presented in Table 1. A total of 160 alleles were identified across 10 breeds assessed with 14 microsatellite loci (Table 1). The number of alleles varied greatly across 14 loci, ranging from only four for MCW098 upto 19 for LEl094. The average number of alleles per locus across 10 breeds was $11.33 \pm 4.9$ (Table 1). This estimate was higher than that reported by other studies on native South African chickens (6.1) (Van-Marle-Koster and Nel, 2000), Zimbabwean ecotypes (9.7) (Muchadeyi et al., 2007; Sabry et al., 2017), Rwanda ecotypes (6.8) (Habimana et al., 2020), native Egyptian and commercial chickens (7.7) (Ramadan et al., 2012), and the Swedish native chicken (4.7) (Abebe et al., 2015). High estimate of the present study could be ascribed to relatively moderate number of loci used in the present study.

The overall mean PIC was $0.72 \pm 0.12$, ranging from 0.45 to 0.88 (Table 1). The mean PIC exceeded 0.5 for six breeds (BAL, DAN, Els, GG, PIR and Sini), which indicated that the panel of microsatellite loci was reasonably informative according to Choi et al. (2015). The lowest PIC across 14 microsatellite loci was 0.37 for the BAL-ISM and TA ecotypes and FAY breed. The PICs in this study (Table 1) were not significantly different from those reported in other studies on native breeds. Ramadan et al. (2012) reported a PIC of 0.65 for six Egyptian native breeds, Seo et al. (2013) reported a PIC of 0.77 for five native Korean lines, and Choi et al. (2015) reported a PIC of 0.68 for commercial Korean native breeds, although all these studies used a large number of markers. However, a lower PIC of 0.55 was reported by Abebe et al. (2015) for five local Swedish breeds; on the other hand, Soltan et al. (2018) recently reported a higher estimate of 0.84 for Sini and Norfa Egyptian native chickens.

The observed proportion of heterozygosity $\left(\mathrm{H}_{\mathrm{obs}}\right)$ is defined as the frequency of heterozygous individuals in the population. For 14 loci across all samples, $H_{\text {obs }}$ averaged 0.46 (Table 1). The expected heterozygosity $\left(\mathrm{H}_{\text {exp }}\right)$ averaged $0.75 \mathrm{H}_{\text {exp }}$ is defined as probability that two gametes randomly select from the gene pool carring different alleles. Both indigenous breeds (BALISM and TA) had the lowest range of $\mathrm{H}_{\text {obs }}$ and $\mathrm{H}_{\text {exp }}$ (Fig. $1 \mathrm{c}, \mathrm{d}$ ), which might be ascribed to the effect of inbreeding. $H_{\text {obs }}$ and $H_{\text {exp }}$ varied lightly across the 14 microsatellite loci (Table 1). All 14 microsatellite loci deviated significantly from HWE $(p<0.01$, results not shown). This variation was the result of various evolutionary mechanisms (mutation, random genetic drift, and/or assortative mating), which unevenly affects various loci (Nei, 1987).

Partitioning of the genetic diversity into within and among populations is best described through Wright $s F_{S t}$ statistics (Wright, 1957). Estimates of Wright $F_{S T}, F_{I T}$ and $F_{\text {IS }}$ are shown in table 2. The means of all three indices were positive. The mean $F_{S T}$ was relatively small $(0.131)$, which indicates similarity of allele frequencies within each breed (Holsinger and Weir, 2009), which in turn indicates that genetic variation among breeds accounted for $13.1 \%$ of the total genetic variation. Furthermore, this small estimate indicates that none of the 14 microsatellite loci were under selection (Excoffier et al., 2009; Sabry et al., 2017). The $F_{\text {ST }}$ values ranged from 0.139 to 0.219 (Table 2) and were higher than those of Ramadan et al. (2012) and Soltan et al. (2018) for native

Table 1: Allele size, number of alleles and polymorphic information content (PIC) values, observed heterozygosity $\left(\mathrm{H}_{\text {obs }}\right.$ ), and expected heterozygosity $\left(\mathrm{H}_{\text {exp }}\right)$ using14 microsatellite markers in10 local chicken strains

\begin{tabular}{llllll}
\hline Marker & Allele size $(\mathbf{b p})$ & Number of alleles & $\mathrm{PIC}$ & $\mathrm{H}_{\text {obs }}$ & $\mathrm{H}_{\text {exp }}$ \\
\hline MCW0206 & $226-240$ & 15 & 0.85 & 0.51 & 0.77 \\
LEI0234 & $217-315$ & 18 & 0.88 & 0.36 & 0.73 \\
MCW0037 & $152-156$ & 12 & 0.67 & 0.32 & 0.66 \\
MCW0222 & $188-254$ & 6 & 0.61 & 0.24 & 0.7 \\
LEI0094 & $254-280$ & 19 & 0.88 & 0.24 & 0.52 \\
MCW0295 & $88-106$ & 10 & 0.70 & 0.59 & 0.89 \\
MCW0098 & $257-324$ & 4 & 0.45 & 0.40 & 0.79 \\
MCW0078 & $135-147$ & 9 & 0.77 & 0.37 & 0.65 \\
MCW0014 & $164-182$ & 15 & 0.68 & 0.54 & 0.87 \\
MCW0183 & $296-326$ & 16 & 0.86 & 0.65 & 0.89 \\
ADL0278 & $114-126$ & 6 & 0.74 & 0.62 & 0.79 \\
MCW0067 & $175-184$ & 6 & 0.65 & 0.50 & 0.72 \\
MCW0216 & $139-149$ & 16 & 0.61 & 0.52 & 0.71 \\
MCW0069 & $158-176$ & & 0.76 & 0.86 \\
\hline
\end{tabular}


Table 2: Wright's fixation indices $\left(F_{S T}, F_{i t}\right.$, and $\left.F_{I S}\right)$ across Taif, Egyptian ecotypes, six Egyptian native chicken and two commercial breeds for 14 microsatellite loci

\begin{tabular}{llll}
\hline Markers & $\mathbf{F}_{s t}$ & $\mathbf{F}_{i t}$ & $\mathbf{F}_{\text {is }}$ \\
\hline ADL278 & 0.138 & 0.151 & 0.340 \\
MCW295 & 0.130 & 0.143 & 0.566 \\
MCW216 & 0.174 & 0.190 & 0.552 \\
MCW014 & 0.194 & 0.211 & 0.678 \\
MCW098 & 0.201 & 0.219 & 0.627 \\
LEI234 & 0.069 & 0.076 & 0.344 \\
MCW078 & 0.218 & 0.237 & 0.439 \\
MCW222 & 0.104 & 0.114 & 0.506 \\
MCW183 & 0.119 & 0.130 & 0.344 \\
LEI094 & 0.100 & 0.109 & 0.226 \\
MCW069 & 0.096 & 0.105 & 0.258 \\
MCW037 & 0.094 & 0.104 & 0.336 \\
MCW067 & 0.064 & 0.071 & 0.362 \\
MCW206 & 0.136 & 0.149 & 0.213 \\
Overallmean & 0.143 & 0.156 & 0.319 \\
\hline
\end{tabular}

Fstatistics were calculated according to Nei (1987) estimations
Egyptian breeds as well as for Korean chickens (Seo et al., 2013). However, Abebe et al. (2015) reported an $\mathrm{F}_{\mathrm{ST}}$ of 0.440 for Swedish local breed, which was higher than our estimate and Habimana et al. (2020) reported that $F_{\text {ST }}$ of 0.610 for Rwanda local breed. The mean global heterozygosity index $\left(F_{I T}\right)$ was 0.143 and ranged from 0.071 to 0.237 (Table 2). This index $\left(F_{I T}\right)$ indicates correlation between alleles within individuals relative to all samples (Holsinger and Weir, 2009). The coefficient of inbreeding $\left(F_{15}\right)$ across 14 microsatellite loci averaged 0.414 and ranged from 0.213 to 0.678 (Table 2); this estimate was higher than those reported in early studies on local chicken breeds. For example, $\mathrm{F}_{1 \mathrm{~s}}$ was 0.018 for Egyptian local breeds (Ramadan et al., 2012), 0.01 for Korean local breeds (Seo et al., 2013) and 0.187 for Swedish breeds (Abebe et al., 2015). However, Soltan et al. (2018) reported a somewhat closer $F_{1 S}$ of 0.41 for the Sini and Norfa local Egyptian chicken breeds. $F_{\text {Is }}$ index is indicative of endangerment and is considered when assessing conservation priorities (Shatokhin et al., 2017). Thus, $F_{\text {Is }}$ value below 0.05 indicates that the breed is not in danger, a value between $0.05-0.15$ indicates the breed is potentially endangered, a value between $0.15-0.25$ indicates it is slightly endangered, a value between $0.25-0.40$ indicates it is endangered, and a value higher than 0.40 shows the breed is considered severely endangered (Ramadan et al.,

Table 3: Estimates of allelic richness (Rt) for each locusTaif, Egyptian ecotypes, six Egyptian native chicken and two commercial breeds

\begin{tabular}{lllllllllll}
\hline Markers & BAL-ISM & Bal & Dan & Els & Fay & GG & PIR & Sini & TA & WL \\
\hline MCW206 & 3 & 7 & 5 & 5 & 5 & 5 & 4 & 6 & 3 & 3 \\
LEI234 & 5 & 12 & 10 & 10 & 4 & 10 & 5 & 11 & 4 & 4 \\
MCW037 & 6 & 3 & 3 & 3 & 3 & 3 & 3 & 3 & 7 & 2 \\
MCW222 & 3 & 4 & 3 & 4 & 4 & 4 & 3 & 4 & 2 & 2 \\
LEI094 & 3 & 11 & 9 & 7 & 8 & 9 & 6 & 10 & 6 & 3 \\
MCW295 & 6 & 12 & 8 & 6 & 5 & 8 & 6 & 11 & 5 & 7 \\
MCW098 & 1 & 2 & 2 & 2 & 2 & 2 & 3 & 2 & 1 & 2 \\
MCW078 & 1 & 4 & 3 & 4 & 3 & 5 & 4 & 5 & 2 & 2 \\
MCW014 & 6 & 4 & 3 & 3 & 1 & 4 & 3 & 3 & 5 & 4 \\
MCW183 & 3 & 9 & 7 & 8 & 5 & 10 & 4 & 9 & 2 & 2 \\
ADL278 & 2 & 5 & 3 & 4 & 2 & 4 & 3 & 5 & 2 & 4 \\
MCW067 & 3 & 3 & 4 & 3 & 3 & 3 & 4 & 3 & 2 & 4 \\
MCW216 & 2 & 3 & 3 & 4 & 3 & 3 & 2 & 3 & 2 & 1 \\
MCW069 & 5 & 8 & 3 & 4 & 5 & 7 & 5 & 6 & 3 & 3 \\
\hline
\end{tabular}

Table 4: Presence of private alleles across Taif, Egyptian ecotypes, six Egyptian native chicken and two commercial breeds

\begin{tabular}{lllllllllll}
\hline Markers & BAL & BAL-ISM & DAN & ELS & FAY & GG & PIR & Sini & TA & WL \\
\hline MCW295 & 1 & 0 & 0 & 0 & 0 & 0 & 0 & 0 & 0 & 0 \\
MCW216 & 0 & 1 & 0 & 0 & 0 & 0 & 1 & 0 & 0 & 0 \\
MCW014 & 1 & 2 & 1 & 0 & 0 & 0 & 0 & 0 & 1 & 0 \\
MCW098 & 0 & 0 & 0 & 0 & 0 & 0 & 1 & 0 & 1 & 0 \\
LEI234 & 1 & 0 & 1 & 0 & 0 & 1 & 0 & 0 & 0 & 0 \\
MCW078 & 0 & 1 & 0 & 0 & 0 & 0 & 0 & 0 & 2 & 0 \\
MCW222 & 0 & 1 & 0 & 0 & 0 & 0 & 0 & 0 & 0 & 0 \\
MCW183 & 0 & 0 & 1 & 0 & 0 & 0 & 2 & 0 & 1 & 1 \\
LEI094 & 0 & 0 & 0 & 0 & 0 & 0 & 1 & 0 & 3 & 1 \\
MCW069 & 3 & 3 & 0 & 0 & 0 & 1 & 0 & 0 & 1 & 0 \\
MCW037 & 0 & 2 & 0 & 0 & 0 & 0 & 0 & 0 & 3 & 0 \\
MCW067 & 0 & 1 & 0 & 0 & 0 & 0 & 0 & 0 & 0 & 1 \\
MCW206 & 1 & 1 & 0 & 0 & 0 & 0 & 1 & 0 & 1 & 0 \\
\hline
\end{tabular}


Table 5: Nei's genetic distance (DA: abovediagonal) and pairwise $F_{S T}$ (below diagonal) estimates of 14 microsatellite loci between each pair of indigenous breeds

\begin{tabular}{lllllllllll}
\hline & BAL-ISM & Bal & Dan & Els & Fay & GG & PIR & Sini & TA & WL \\
\hline BAL-ISM & & 0.77 & 0.76 & 0.79 & 0.76 & 0.72 & 0.77 & 0.73 & 0.33 & 0.73 \\
Bal & 0.32 & & 0.13 & 0.08 & 0.10 & 0.06 & 0.50 & 0.04 & 0.81 & 0.47 \\
Dan & 0.33 & 0.10 & & 0.17 & 0.23 & 0.16 & 0.47 & 0.14 & 0.77 & 0.52 \\
Els & 0.33 & 0.08 & 0.12 & & 0.18 & 0.06 & 0.52 & 0.06 & 0.81 & 0.51 \\
Fay & 0.37 & 0.11 & 0.17 & 0.15 & & 0.15 & 0.56 & 0.13 & 0.82 & 0.51 \\
GG & 0.31 & 0.08 & 0.12 & 0.08 & 0.14 & & 0.49 & 0.04 & 0.75 & 0.49 \\
PIR & 0.33 & 0.23 & 0.24 & 0.25 & 0.30 & 0.24 & & 0.48 & 0.86 & 0.33 \\
Sini & 0.29 & 0.06 & 0.10 & 0.08 & 0.12 & 0.07 & 0.22 & & 0.78 & 0.47 \\
TA & 0.26 & 0.33 & 0.34 & 0.34 & 0.39 & 0.32 & 0.36 & 0.31 & & 0.81 \\
WL & 0.31 & 0.22 & 0.24 & 0.25 & 0.28 & 0.24 & 0.20 & 0.21 & 0.36 & \\
\hline
\end{tabular}

2012; Soltan et al., 2018). Fig. 3 shows $F_{\text {IS }}$ for each of the 10 breeds. It was high for all breeds, with BAL-ISM and local TA breeds having highest values (0.756 and 0.742$)$. Using 20 microsatellite loci, Soltan et al. (2018) reported $F_{\text {is }}$ of 0.451 for the Sini breed, which was higher than our estimate (0.216). High $\mathrm{F}_{\mathrm{IS}}$ reported in the present study, as well as other studies, indicates of taking immediate breeding and conservative measures to preserve these endangered breeds.

As the observed number of alleles in a sample depends on the sample size, $R_{t}$ was estimated, which is defined as the mean number of alleles per locus (Hughes et al., 2008). The

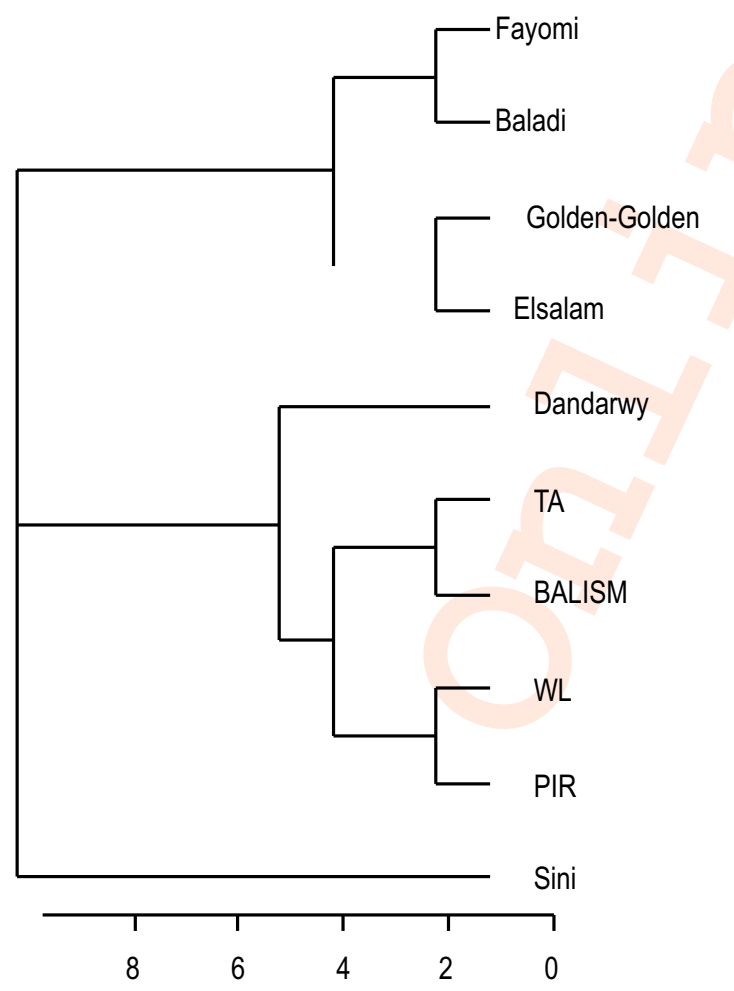

Fig.1: Neighbor-joining tree of Taif, Egyptian ecotypes, six Egyptian native chicken and two commercial breeds using 14 microsatellites loci. importance of $R_{t}$ is that a decline in $R_{t}$ can prompt a decrease in the population's ability to adjust to future ecological change, as this type of diversity is the crude material for evolutionary forces such as natural selection (Ramadan et al., 2012). The estimates of $R_{t}$ presented in Table 3 were variable, ranging from 1 to 12 with an average of 4.4. Few studies have utilized $R_{t}$ as a measure of genetic diversity; high $R_{t}$, even for neutral alleles, enhances evolvability by enlarging the fraction of genotypic space accessible to mutational events. Therefore, if no allelic richness exists, no standing variation exists (Wagner, 2008). Consequently, $R_{t}$ is a solid indicator of the capability of a population to evolve, and it has been concluded that this measure is important for population conservation and management (Caballero and Garcia-Dorado, 2013). The estimates of $R_{t}$ for the BAL-ISM and TA ecotypes ranged from 1 to 6 and 1 to 7 , respectively, which indicates shrinking evolvability of both ecotypes. This result also supports the conclusion derived from the $F_{\text {Is }}$ values. Private alleles were found for all 14 microsatellite loci (Table 4).

The highest number of private alleles was 13 for TAecotype whereas no private alleles were detected for Els, FAY and Sini breeds. Among the 14 studied microsatellite loci, the marker MCW069 showed the largest number of private alleles (eight) whereas MCW014, MCW183 and MCW037 had five private alleles. These results indicate diversification of selective pressures acting on specific alleles. Nei's genetic distance (DA) and $F_{S T}$ were calculated for 10 chicken breeds over 14 microsatellite loci (Table 5). The results showed that DA averaged 0.46 and the smallest distance was 0.04 between BAL and Sini breeds.

This result was supported by high percentage of shared alleles (80\%) between these breeds (Table 5) whereas the largest distance of 0.86 was found between TA ecotype and PIR breed, which shared the lowest percentage of alleles. The smallest $F_{S T}$ was found between BAL and Sini breeds (0.06), while the highest (0.39) was noted between FAY and TA ecotypes, followed by PIR and TA ecotype (0.36) (Table 5). These results were consistent with the DA results. Fig.1 shows the neighborjoining (NJ) phylogenetic tree (Saitou and Nei, 1987) for 10 breeds. NJ phylogenetic tree was divided into three large clades. The first clade consisted of two lineages, which 


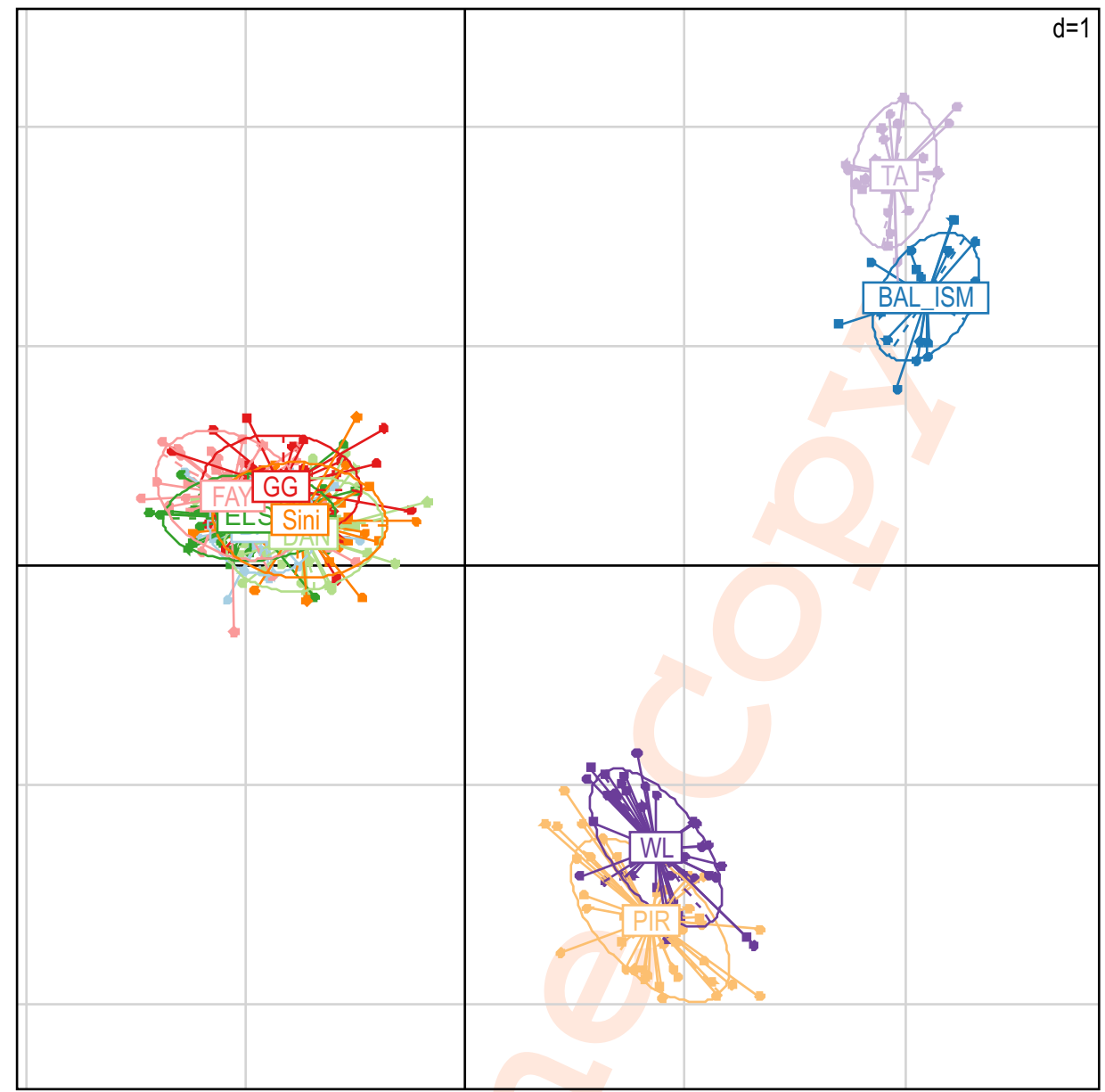

Fig. 2: Principle component analysis of Taif, Egyptian ecotypes, six Egyptian native chicken and two commercial breeds across 14 microsatellites loci.

comprised four Egyptian indigenous breeds, and FAY and BAL in the first lineage, and GG and Els in the second lineage. The second clade was divided into three lineages, where two ecotypes occupied one lineage, and two pure breeds occupied the second, while DAN constituted the third lineage. A similar phylogenetic tree was reported by Ramadan et al. (2012). This result is in accordance with the findings of Osaman et al. (2016), who used complete sequence of mitochondrial DNA D-loop region to prove that the western Asian native chicken clustered together with the Egyptian native chicken breeds.

The last solely clade comprised of Sini breed. A similar phylogenetic tree was reported by Osaman et al. (2016). Principal component analysis (PCA) is presented in Fig. 2. The results of the analysis reflect a similar finding to the phylogenetic analysis. Both ecotypes grouped together, while six Egyptian breeds constituted another group, and pure breeds of chicken were grouped together. The difference between PCA and NJ phylogenetic analyses might be a result of differences in the algorithm used in each method.
In conclusion, the results of the present study revealed that the two ecotypes appeared to be genetically correlated, have an endangered status and limited allele richness, which is indicative of the shrinking evolvability of studied ecotypes. Lower genetic distance and $F_{S T}$ values were reported for the Egyptian indigenous breeds. Phylogenetic analysis showed that the Dandarawy native Egyptian chicken breed was genetically closest to both Egyptian and Saudi ecotypes.

\section{Acknowledgment}

The authors extend their appreciation to Taif University for funding current work under Research Supporting Project Number(TURSP-2020/59), Taif University, Taif, Saudi Arabia.

\section{Add-on Information}

Authors' contribution: A. Sabry and S. Ramadan: Formal analysis and Methodology; M.M. Hassan and A.A.Mohamed: Writing-original draft and Laboratory work; A. Mohammedein 
and M. Inoue-Murayama: Writing, review \& editing and funding current work.

Research content: The research contents is original and has not been published elsewhere

Ethical approval: The procedures and protocols used in this study were designed in accordance with the guidelines for animal welfare and the use of animals prepared by the Institutional Animal Care and Ethical Committee of Taif University, Saudi Arabia for project TURSP-2020/59.

Conflict of interest: The author declares that there is no conflict of interest.

Data from other sources: NotApplicable.

Consent to publish: All authors agree to publish the paper in Journal of Environmental Biology.

\section{References}

Abdel-Alah, M.H., M.M. Iraqi, M.H. Khalil, G.M. El-Moghazy and M.M. ElAtrouny: Quantitative trait loci associated with egg traits in F2 Intercross between Golden Montazah and White leghorn chickens. Benha J. Appl. Sci., 2, 1-10 (2017).

Abebe, A.S., S. Mikko and A.M. Johansson: Genetic diversity of five local Swedish chicken breeds detected by microsatellite markers. PLOS ONE, 10, e0120580 (2015).

Caballero, A. and A. Garcia-Dorado: Allelic diversity and its implications for the rate of adaptation. Genetics, 195, 1373-1384 (2013).

Choi, N.R., D.W. Seo, S.B. Jemaa, H. Sultana, K.N. Heo, C. Jo and J.H. Lee: Discrimination of the commercial Korean native chicken population using microsatellite markers. J. Anim. Sci. Technol., 57, 5-12 (2015).

Dwivedi, A.K. and B.D. Tripathi: Pollution tolerance and distribution pattern of plants in surrounding area of coal-fired industries. $J$. Environ. Biol., 28, 257-263 (2007).

Excoffier, L., T. Hofer and M. Foll: Detecting loci under selection in a hierarchically structured population. Heredity, 103, 285-298 (2009).

Fathi, M.M., I.Al-Homidan, M.I. Motawei, O.K.Abou-Emera and M.F. El-Zarei: Evaluation of genetic diversity of Saudi native chicken populations using microsatellite markers. Poult. Sci., 96,530-536 (2017).

Food and Agriculture Organization of the United Nations: Molecular Genetic Characterization of Animal Genetic Resources. FAO Animal Production and Health Guidelines. No. 9. Food and Agriculture Organization of the United Nations, Rome, Italy (2011).

Habimana, R., T.O. Okeno, K. Ngeno, S. Mboumba and P. Assami: Correction, genetic diversity and population structure of indigenous chicken in Rwanda using microsatellite markers. PLOS ONE, 15, e0238966(2020).

Hillel, J., M.A. Groenen, M. Tixier-Boichard, A.B. Korol, L. David, V.M. Kirzhner, T. Burke, A. Barre-Dirie, R.P. Crooijmans, K. Elo, M.W. Feldman, P.J. Freidlin, A. Mäki-Tanila, M. Oortwijn, P. Thomson, A. Vignal, K. Wimmers and S. Weigend: Biodiversity of 52 chicken populations assessed by microsatellite typing of DNA pools. Genet. Sel. Evol., 35, 533-557 (2003).

Holsinger, K.E. and B. Weir: Genetics in geographically structured populations: Defining, estimating and interpreting $\mathrm{F}_{\mathrm{ST}}$. Nat. Rev. Genet.,10,639-650 (2009).

Huang, S., L. Zhang, M.U. Rehman, M.K. Iqbal, Y. Lan, K. Mehmood, H.
Zhang, G. Qiu, F. Nabi, W. Yao, M. Wang and J. Li: High altitude hypoxia as a factor that promotes tibial growth plate development in broiler chickens. PLOS ONE, 27, e0173698 (2016).

Hughes, R.A., B.D. Inouye, M.T.J. Johnson, N. Underwood and M. Vellend: Ecological consequences of genetic diversity. Ecol. Lett., 11,609-623 (2008).

Hwangbo, J., E.C. Hong, A. Jang, H.K. Kang, J.S. Oh, B.W. Kim and B.S. Park: Utilization of house fly-maggots, a feed supplement in the production of broiler chickens. J. Envir. Biol., 30, 609-614 (2009).

Jombart, T.: Adegenet: A R package for the multivariate analysis of genetic markers. Bioinformatics, 24, 14035 (2008)

Muchadeyi, F.C., H. Eding, C.B. Wollny, E. Groeneveld, S.M. Makuza, R. Shamseldin, H. Simianer and S. Weigend: Absence of population substructuring in Zimbabwe chicken ecotypes inferred using microsatellite analysis. Anim. Genet., 38, 332-339 (2007).

Nei, M.: Molecular Evolutionary Genetics. Columbia University Press, New York, NY, USA(1987).

Nones, K., M.C. Ledur, D.C. Ruy, E.E. Baron, A.S.A.M.T. Moura and L.L. Coutinho: Genetic linkage map of chicken chromosome 1 from a Brazilian resource population. Sci. Agric., 1, 12-17 (2005).

Osaman, S.A.M., T. Yonezawa and M. Nishibori: Origin and genetic diversity of Egyptian native chicken based on complete sequence of mitochondrial DNA D-loop region. Poult. Sci., 95, 1248-1256 (2016).

R Core Team.: R:A Language and Environment for Statistical Computing. R Foundation for Statistical Computing, Vienna, Austria (2020).

Ramadan, S., B.B. Kayang, E. Inoue, K. Nirasawa, H. Hayakawa, S. Ito and $\mathrm{M}$. Inoue-Murayama: Evaluation of genetic diversity and conservation priorities for Egyptian chickens. Open. J. Anim. Sci., 2,183-190 (2012).

Rudresh, B.H., H.N.N. Murthy, M.R. Jayashankar, C.S. Nagaraj, A.M. Kotresh and S.M. Byregowda: Microsatellite based genetic diversity study in indigenous chicken ecotypes of Karnataka. Vet. World, 8, 970-976 (2015)

Sabry A., M.M. Hassan, A.A. Mohamed, H.H. Allam and A. Gaber: Quantitative assessment of genetic diversity among local chicken breeds detected by microsatellite markers. Biosci. Res., 14, 900907 (2017)

Saitou, N. and M. Nei: The neighbor-joining method: A new method for reconstructing phylogenetic trees. Mol. Biol. Evol., 4, 406-425 (1987).

Sanusi, A.R. and S.O. Oseni: Nigerian Fulani ecotype chickens: Growth performance under two production systems. Gen. Biodiv. J., 4, 14$21(2020)$.

Seo, D.W., M.R. Hoque, N.R. Choi, H. Sultana, H.B. Park, K.N. Heo, B.S. Kang, H.T. Lim, S.H. Lee, C. Jo and J.H. Lee: Discrimination of Korean native chicken lines using fifteen selected microsatellite markers. Asian-australas. J. Anim. Sci., 26, 316-322 (2013).

Shafey H.I., M.D. Aboelhassan, E.M. EL-Komy, R.E. El-karim and K.F. Mahrous: SNP of cGH gene in Egyptian chicken breeds at Mspl site. Biosci. Biotech. Res. Asia, 14, 33-41 (2017).

Shatokhin, Y., M. El Gammal and D. Prikhodko: Arab Republic of Egypt. Broiler poultry industry: Investment challenges and opportunities (Draft for discussion). Food and Agriculture Organization of the United Nations, Rome, Italy, pp. 5-43 (2017).

Soltan, M., S. Farrag, A. Enab, E. Aboü-Elewa, S. El-Safty and A. Abushady: Sinai and Norfa chicken diversity revealed by microsatellite markers. S. Afr. J. Anim. Sci., 48, 307-315(2018).

Van Marle-Koster, E. and L.H. Nel: Genetic characterization of native southern African chicken populations: evaluation and selection of polymorphic microsatellite markers. S. Afr. J. Anim. Sci., 30, 1-6 (2000).

Wagner, A.: Robustness and evolvability: A paradox resolved. Proceedings of the Royal Society B: Biol. Sci., 275, 91-100 (2008).

Wright, S.: The genetical structure of populations. Ann. Eugen., 15, 323354 (1951) 